\title{
THE DISTRIBUTION OF PARTICLES IN THE WALL REGION OF THE CYCLONE CHAMBER
}

\author{
Aleksandr Razva ${ }^{1,}$, Michail Vasilevsky ${ }^{1}$, Alice Pleshco ${ }^{1}$, and Aleksandr Naumkin ${ }^{1}$ \\ ${ }^{1}$ National research Tomsk polytechnic university, 634050 Tomsk, Russia
}

\begin{abstract}
Held cyclone operation analysis at different flow rates. In the absence of dust deposits on the surface of the device in countercurrent conical cyclone particle concentration distribution in the circumferential direction in the wall region is uneven rope-formed particles to form clots. At low concentrations where clots stably transported stream at any position of the cyclone can be made based on evaluation of the speed distribution of gas and particles in the bunches movement. In work the analysis of work of a cyclone is carried out at various speeds of a stream.
\end{abstract}

\section{Introduction}

Processes of a current of the two-phase environment in the cyclone chamber are difficult and still there is no physically reasonable model of transformation of the disperse environment from an aerosol state in bulk in the receiver. At the boundary of the swirling flow near the boundary surface of gas flow tangential speed decreases and becomes zero at the wall of the flow itself. Thus, the particles are near the wall in dynamic equilibrium at the interface and the transfer of particles flow in a radial direction on average no (condition not pickup particles) [1].

In the absence of dust deposits on the device surface in a conical counter-cyclone in cinders-output hole concentration of particles increases hundreds of times, causing the flow of braking due to the increased value of the shear stress on the wall. Bundles already formed on $1 / 4$ traffic from the injection point of the particles at high concentrations [2]. For larger particle concentrations at which the weight of bundles becomes sufficient for the manifestation of the forces of gravity, calculation of flow characteristics are shown in [3]. At low concentrations, at which the bundles stably transported cyclone flow at any position can be made based on evaluation of the speed distribution of gas and particles in the harness movement.

\footnotetext{
* Corresponding author: razva@mail.ru
} 


\section{The distribution of particles in the wall region of the cyclone chamber}

To determine the time of passage of the material through the cyclone and comparing it with a gas residence time were conducted measuring the air speed at the inlet of the entire apparatus during retraction cinders-output air through the hole, flow of particulate material. Conducted immediate disconnection from the cyclone collector vacuum, determined the amount of dust precipitated in the cyclone. [4] Visually it was evident (figure 1), that the dust in the cone moves to a bandpass harnesses.

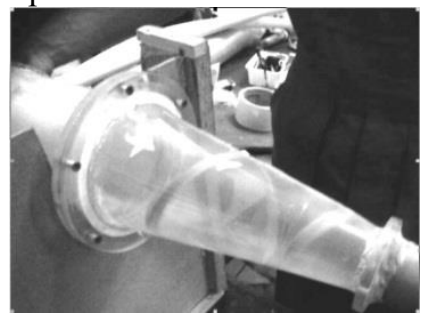

Fig. 1. The configuration of the dispersed phase in the cyclone cone.

The number of turns, their length was determined visually, as well as the calculation. The air speed in the turns was determined taking into account the acceleration of the flow in the cone, and averaged over the length of the coil. Determined during the passage of air through the cyclone cone as the cone volume divided by the flow rate, and while loop length divided by the tow speed. We determined the residence time, as the mass is left out in the cyclone with a cut-off the flow divided by the mass flow rate. Experiments were made on the powder M40 (corundum). Consumption was $1,1 \mathrm{~g} / \mathrm{s}$, flow rate $9-17 \mathrm{~g} / \mathrm{s}$, the speed of air in the inlet section $7-13 \mathrm{~m} / \mathrm{s}$. The ratio of the time of passage of the material to the air passage of time depending on the concentration and speed of air is in the range 2 $\div 30[4]$.

In order to determine the parameters of clotting (dust distribution M40 in the circumferential direction) in a countercurrent cyclone via the rotary section, air samples were taken at different concentrations of particles (or the number of allocated dust on the amount of the introduced dust) on the circumference of the cyclone at a fixed height equal to 1.5 the diameter of the input. It was found that concentration distribution is uneven in the circumferential (and concentrations are burst gaps) (figure 2).

We will enter coefficient of unevenness of distribution of particles in the district direction $\mathrm{Ku}=\mathrm{qmax} / \mathrm{qmin}$. At concentration of dust $2-4 \mathrm{~g} / \mathrm{m}^{3}$ monofractional dust $\mathrm{Ku} \approx 3$, at concentration of the same dust $70-95 \mathrm{~g} / \mathrm{m}^{3}$ in an entrance stream $\mathrm{Ku} \approx 5$. For polyfractional dust with concentration $11-19 \mathrm{~g} / \mathrm{m}^{3} \mathrm{Ku} \approx 7$. At concentrations of dust more $200 \mathrm{~g} / \mathrm{m}^{3}$ uneven particle distribution shown in circumferentially tows, which move with low speed in the circumferential direction and are detected visually. The computational model of concentration of particles in countercurrent cylindrical cyclone device disclosed in [1]. 
Cylinder

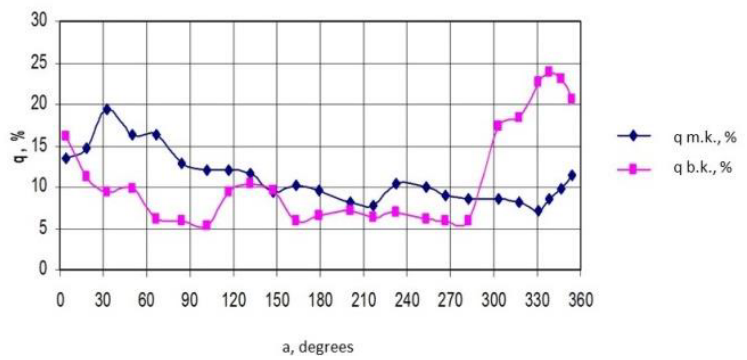

Fig. 2. The relative amount of monofractional dust output from the periphery of cylindrical part of a cyclone by $15 \%$ amount of air depending on angular coordinate (the direction of counting in the direction of rotation of a stream, a reference mark interface of input to the chamber cylinder): concentration 2-4 g/ $\mathrm{m}^{3}$, - concentration $70-95 \mathrm{~g} / \mathrm{m}^{3}$.

The relative yield of cement dust is presented in figure 3 .
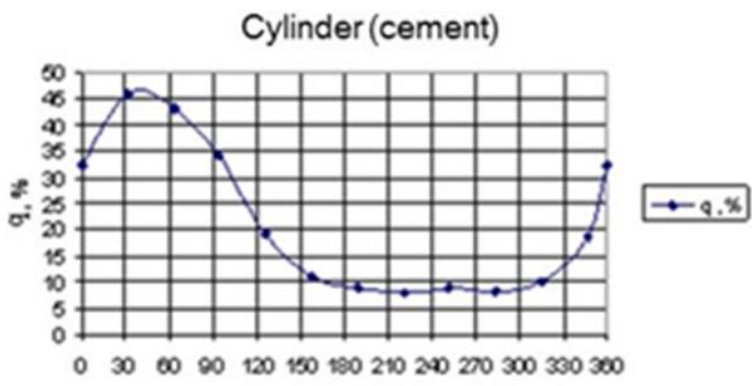

a, degrees

Fig. 3. The relative amount of polyfractional dust output from the periphery of cylindrical part of a cyclone by $15 \%$ amount of air depending on angular coordinate (the direction of counting in the direction of rotation of a stream, a reference mark interface of input to the chamber cylinder): concentration $11-19 \mathrm{~g} / \mathrm{m}^{3}$.

\section{Estimates of parameters tows at high particle concentrations}

Let $C_{*}-$ the absolute speed harness (figure 4), then $C_{*} \cos \alpha-$ the circumferential speed of the harness, $\alpha$ - the angle between the $C_{*}$ and district, $\ell$ - harness length, $\mathrm{t}-$ while its location on the surface, $t=\ell / C_{*}$, Wח - dust flow, $\mathrm{m}-$ harness weight, $\eta_{*}=m / W_{\Pi} t_{-}$ coefficient of dust into a bundle.

Length of a plait is equal to length of one round increased by number of rounds $\ell=\frac{\pi D}{\cos \alpha} \frac{H}{\pi D \operatorname{tg} \alpha}=\frac{H}{\sin \alpha}$

where $\mathrm{H}$ - height of separation part where the plait moves, $\mathrm{D}=2 \mathrm{R}-$ diameter of separation part, $m=A_{\mathrm{S}} \ell(1-\varepsilon) \rho_{\delta} ; A_{\mathrm{S}}=\pi r_{\mathrm{S}}^{2}$ - plait cross-sectional area, $\varepsilon-$ the fractional void volume porosity of the dust in a plait, $\rho_{\delta}-$ dust density, $W_{\Pi}=\rho K_{\text {in }} \mathrm{Q}=m C_{*} / \ell \eta_{*} ; m=\rho K_{\text {in }} Q \ell \eta_{*} / C_{*}$. In the circumferential direction of the friction force on the tow surface is balanced by the wind carrying away the power flow: 


$$
\frac{m C_{*}^{2} \cos ^{2} \alpha}{R} \varphi_{\mathrm{S}}=\xi_{\mathrm{S}} r_{\mathrm{S}} H \frac{\rho}{2}\left(V_{\varphi}-C_{*} \cos \alpha\right)^{2}
$$

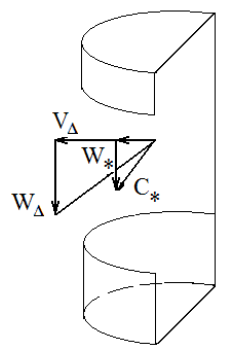

Fig. 4. The scheme of distribution of speed of a plait in the cyclonic device.

Here $\varphi_{\mathrm{S}}, \xi_{\mathrm{S}}$ - coefficients of friction of particles about a surface and the aerodynamic resistance of a plait, $V_{\varphi}$ - district speed of gas at distance from a wall $y=r_{S}$, $\mathrm{V}_{\varphi}(\mathrm{y})=\sqrt{\frac{\tau_{w}}{\rho}}(9.5+1.32 \ln \eta) ; \eta=\frac{y}{v} \sqrt{\frac{\tau_{\mathrm{W}}}{\rho}}[8], \tau_{\mathrm{w}}=0.5 C_{\mathrm{f}} \rho V_{\Delta}^{2} / 2, C_{f} \approx 0.006 ; V_{\Delta}-$ value of district speed of gas on border of a wall zone; $V_{\Delta}=0.95 V_{\text {in }}$.

Tow speed in the axial direction $W_{*}=W_{\Delta}\left(r_{S} / \Delta\right)^{1 / 14}$, here $W_{\Delta}-$ harness the maximum speeds at a distance from the wall $y=\Delta ; \Delta$ - distance border flow core and the wall region [5]. Substituting $C_{*}=\frac{W_{*}}{\sin \alpha}=\frac{W_{\Delta}}{\sin \alpha}\left(\frac{r_{\mathrm{S}}}{\Delta}\right)^{1 / 14}$ in (1) and considering that $r_{S} / R=\left(r_{S} / \Delta\right)(\Delta / R)$ and designating $(9.5+1.32 \ln \eta)=\eta_{\mathrm{y}}$, after transformations we obtain

$$
\left[\frac{\varphi_{S}}{\xi_{S}} \frac{2 \pi \Delta}{\mathrm{R}} \frac{(1-\varepsilon) \rho_{\delta}}{\rho}\right]^{1 / 2} \frac{\mathrm{W}_{\Delta}}{\mathrm{V}_{\Delta}}\left(\frac{\mathrm{r}_{S}}{\Delta}\right)^{8 / 14}=\sin ^{1 / 2} \alpha\left[\eta_{y} \operatorname{tg} \alpha-\frac{\mathrm{W}_{\Delta}}{\mathrm{V}_{\Delta}}\left(\frac{\mathrm{r}_{S}}{\Delta}\right)^{1 / 14}\right]
$$

When converting into account that $H / \ell=\sin \alpha$. Meaning $\Delta \approx 0.03 R \quad$ и $\varepsilon=0.7 \ldots 0.95$ correspond to the size of the wall region and the porosity of particles in a bundle.

From the relation $\pi r_{\mathrm{S}}^{2}(1-\varepsilon) \rho_{\delta} \ell=\rho K_{\mathrm{in}} Q \ell \eta_{*} / C_{*}$ find the value of $r_{S}$.

$$
r_{\mathrm{S}}=\sqrt{\frac{\rho}{\rho_{\delta}} \frac{K_{\mathrm{in}} V_{\mathrm{in}} f_{\mathrm{in}} \eta_{*}}{(1-\varepsilon) \pi C_{*}}}
$$

here $Q=V_{\text {in }} f_{\text {in }} ; V_{\text {in }}, f_{\text {in }}$ - stream speed in the entering branch pipe, the section of a branch pipe through passage. The equations $(2,3)$ allow to define $C_{*}$ и $r_{S}$. Estimates show that at low concentrations $K_{\text {in }}=(1 \ldots 10) \cdot 10^{-4} \mathrm{~kg} / \mathrm{kg}$ and a flow of less than 5 micron particles, the quantity $r_{S}$ it is $100 \ldots 300 \mathrm{~mm}$, that particle size reaches effectively released from the stream at the receiver. Thus, the reason for trapping fine particles in the cyclone - binding bundles and their transportation in the receiver rope-formed state. In very small 
concentrations of fine particles occurs probably periodic accumulation of particles in the volume and precipitation cord factor receiver. In this case there are pressure pulsations in the receiver. In [2] provides information about the flow of brake particles in the harness.

\section{Conclusion}

The swirling flow efficiency of dust formed from bundles depends on the degree of dispersion in the dust receptacle harnesses gas flows. The flow of the cyclone effectively work towards opening dust-collecting bag wasting their energy on the generation of the second kind of vortices and the harness transport. For the dispersion of the bundles in the receiver must also be expended energy. This energy will be low if the receiver will continue cord factor process and attenuation flow twist. The cyclone receiver individually, depending on its design, the generation of turbulence is the nature and intensity gradient it is insufficient to break the harness. In receivers such as inertial relief in which turbulence damping occurs not only, but also increases the expense of additional vortex formation, separation of fine particles is not possible due to dispersion harnesses. The same situation is multicyclones receivers in which holes exiting dust-collecting bag torches interact and as a result, turbulence intensity in the common dust collecting chamber is comparable to the intensity of turbulence in the flame. This fact can be explained by the low efficiency of gas dedusting cyclone dust collector. [2] The most radical solution to the problem of increasing the stability and efficiency of cyclone systems is the use of transit "receivers", in which there is a flow attenuation and naturally formed layer of particles [2].

\section{Acknowledgments}

The work was held within the research state assignment "Science" No. 13.1339.2014/K (Code of Federal Target Scientific and Technical Program 2.1410.2014).

\section{References}

1. M.V. Vasilevsky, E.G. Zykov., and A.S. Razva. Theor. Found. Chem. Eng. 45, 3 (2011)

2. M.V. Vasilevsky Dedusting gas inertial devices (Publishing house of Tomsk Polytechnic University, Tomsk, 2008)

3. V. Strauss. Industrial cleaning gases, (Chemistry, Moscow, 1981)

4. M. V. Vasilevsky, V.I. Romandin, E. G. Zykov, V.A. Polyushko., A. S. Razva. Bulletin of the Tomsk State University. Mathematics and Mechanics 23, 3 (2013)

5. A.N. Shtym. Aerodynamics of cyclonic swirl chambers (Publishing house of Far East university, Vladivostok, 1985) 